\title{
Effect of Mangosteen Pericarp Extract on Gastric Mucosal Damage Induced by Aspirin
}

\author{
Jansen Budiono, ${ }^{1}$ Achadiyani, ${ }^{2}$ Dolvy Girawan ${ }^{3}$ \\ ${ }^{1}$ Faculty of Medicine, Universitas Padjadjaran, ${ }^{2}$ Department of Anatomy and Cell Biology Faculty \\ of Medicine Universitas Padjadjaran, ${ }^{3}$ Department of Internal Medicine Faculty of Medicine \\ Universitas Padjadjaran/Dr. Hasan Sadikin General Hospital Bandung
}

\begin{abstract}
Background: Peptic ulcer disease is commonly found in the community. Nonsteroidal anti-inflammatory drug (NSAID) such as aspirin is one of the predisposing factors to ulcers. According to various studies, plant extracts have been shown to produce promising results in the treatment of peptic ulcers. Xanthone, the active substance contained in the mangosteen pericarp extract has been extensively studied for its role in various diseases. The present study was undertaken to identify the effect of mangosteen pericarp extract on gastric mucosal damage.

Methods: This study was carried out from September-November 2014 in the Animal Laboratory of the Department of Pharmacology and Therapy, Faculty of Medicine, Universitas Padjadjaran/Dr. Hasan Sadikin General Hospital, Bandung. Thirty two rats were randomly divided into 2 groups with different treatments and were induced by aspirin. Histologic studies were conducted to determine the score of gastric mucosal damage. The parameters used were Wattimena's criteria for damage of gastric mucosa. Data was analyzed using Chi Square test and the outcome was measured in relative risk.

Results: The study revealed a significant association between pretreatment with mangosteen pericarp extract and incidence of gastric mucosal damage induced by aspirin $(\mathrm{p}<0.05)$. Calculation with a modified $2 \times 2$ table revealed a relative risk of 0.625 .

Conclusions: Rats pretreated with mangosteen pericarp extract exhibit less gastric mucosal damage, in experimentally aspirin-induced ulcer. [AMJ.2016;3(3):388-91]
\end{abstract}

Keywords: Aspirin, gastric mucosal damage, mangosteen pericarp extract

\section{Introduction}

Peptic ulcer is commonly found in the community. Its impact has been substantial proven to increase morbidity, and serious complications lead to mortality.

Nonsteroidal anti-inflammatory drug (NSAID) such as aspirin is one of the predisposing factors to ulcers. ${ }^{1}$ Unfortunately, Aspirin and NSAIDs are among the most frequently used drugs worldwide. ${ }^{2}$ Prevention of peptic ulcer due to NSAID use is an important clinical issue.

Furthermore, herbal medicine is still widely used until presently due to better cultural acceptability. According to various studies, plant extracts have been shown to produce promising results in the treatment of peptic ulcers. ${ }^{3}$ Mangosteen pericarp extract which contained xanthone as its active substance, has now become widely available as a nutraceutical (or food supplements) and further studies on its therapeutic benefits would considerably benefit the general population. ${ }^{4}$

The present study was conducted to identify the effect of mangosteen pericarp on gastric mucosal damage.

\section{Methods}

This animal experimental study was carried out from September-November 2014 in the Animal Laboratory of Department of Pharmacology and Therapy, Faculty of Medicine, Universitas Padjadjaran/Dr. Hasan Sadikin General Hospital, Bandung. Experimental protocols had been approved by the Health Research Ethics Committee of the 
Faculty of Medicine, Universitas Padjadjaran.

Mangosteen pericarp extract in this study employed the standardized herbal Mastin. Mastin was purchased from drug stores in Bandung, Indonesia.

Thirty two adult male rats (Rattusnorvegicus), Wistar-strain, age 2-3 months, weighing 175-200g were used as subjects. The Federer formula was used to calculate the number of rats. Meanwhile, the adaptation performed in this study was based on the protocol used by Nainwal et al. ${ }^{5}$ The rats were housed under the same conditions and fed with the standard chow diet and water ad libitum for 2 days before the study, maintained with light/dark cycle of $12 / 12$ hours in room temperature. The exclusion criteria were weight loss $>10 \%$ during adaptation period.

After the adaptation period, the rats were randomly divided into 2 groups of 16 rats each. Group I (control group) received $4 \mathrm{ml}$ corn oil (vehicle) orally for 7 consecutive days. Group II (pretreated group) received pretreatment with mangosteen pericarp extract $(500 \mathrm{mg} /$ $\mathrm{kg}$ body weight) suspended in $4 \mathrm{ml}$ corn oil orally for 7 consecutive days. The mangosteen pericarp extract dosage was determined by Nainwal et al. ${ }^{5}$

Furthermore, at the end of the study, the rats fasted for 24 hours. Thirty minutes after the last treatment; the rats received aspirin $(200 \mathrm{mg} / \mathrm{kg}$ body weight). The duration of treatment and aspirin dosage for the above mentioned groups were sufficient to induced gastric mucosal damage as showed by a study performed by Jainu et al. ${ }^{3}$ After 4 hours of aspirin induction, the rats were sacrificed under diethyl ether anesthesia; then the stomach was dissected out and processed for histologic preparation.

The histologic study was carried out at the Department of Anatomy-Biocellular, Faculty of Medicine, Universitas Padjadjaran by using the light microscope. Each rat specimen was analyzed under 10 high-power fields (HPF). Then, each group was analyzed with 160 fields and the total of fields examined was 320 . The parameter used was Wattimena's ${ }^{6}$ criteria for damage of gastric mucosa as follows: 1) Score 1: no erosion, 2)Score 2: erosion was limited to surface epithelium, 3)Score 3: erosion was extended to 1/3 upper gastric gland, 4)Score 4: erosion was extended to $1 / 3$ middle gastric gland, 5)Score 5: erosion was extended to $1 / 3$ lower gastric gland, 6)Score 6: erosion with extension to muscularis mucosae.

The association between two variables was tested using chi-square test. The nullhypothesis would be accepted if $p$-value $<0.05$. Should there be a significant association between the two variables; the relative risk for the modified category would then be calculated. Score 1 was categorized as no erosion while scores 2-6 were categorized as erosion.

\section{Results}

Incidence of gastric mucosal damage induced by aspirin in each group was varied (Table 1).

The results showed significant relationship between pretreatment with mangosteen pericarp extract and incidence of gastric mucosal damage induced by aspirin ( $\mathrm{p}$-value: 0.000 ). The data was then modified to fit a $2 \times$ 2 table for relative risk calculation (Table 2). The calculation then revealed a relative risk of 0.625 .

\section{Discussions}

Furthermore, the result based in Table 1 shows that there was significant association between two variables. However, this result couldnot explain which group exhibited little or extensive difference in incidence of gastric mucosal damage. Relative risk calculation could determine whether mangosteen pericarp extract could provide protection or adverse effects. Modification of Table 1 to fit a $2 \times 2$ table was necessary in order to calculate relative risk. Additionally, calculation based on Table 2 revealed a relative risk of 0.625

Table 1 Score of Gastric Mucosal Damage in Each Group

\begin{tabular}{|c|c|c|c|c|c|c|c|c|c|c|c|c|c|c|}
\hline \multirow{3}{*}{ Group } & \multicolumn{12}{|c|}{ Gastric Mucosal Damage Score } & \multirow{3}{*}{ Total } & \multirow{3}{*}{$\%$} \\
\hline & \multicolumn{2}{|c|}{1} & \multicolumn{2}{|c|}{2} & \multicolumn{2}{|c|}{3} & \multicolumn{2}{|c|}{4} & \multicolumn{2}{|c|}{5} & \multicolumn{2}{|c|}{6} & & \\
\hline & $\mathbf{n}$ & $\%$ & $\mathbf{n}$ & $\%$ & $\mathbf{n}$ & $\%$ & $\mathbf{n}$ & $\%$ & $\mathbf{n}$ & $\%$ & $\mathbf{n}$ & $\%$ & & \\
\hline I & 24 & 15 & 45 & 28.1 & 23 & 14.4 & 24 & 15 & 16 & 10 & 28 & 17.5 & 160 & 100 \\
\hline II & 78 & 48.8 & 37 & 23.1 & 23 & 14.4 & 8 & 5 & 9 & 5.6 & 5 & 3.1 & 160 & 100 \\
\hline Total & 102 & 31.9 & 82 & 25.6 & 46 & 14.4 & 32 & 10 & 25 & 7.8 & 33 & 10.3 & 320 & 100 \\
\hline
\end{tabular}


Table 2 Modified Table for Relative Risk Calculation

\begin{tabular}{|c|c|c|c|c|c|c|}
\hline \multirow{3}{*}{ Group } & \multicolumn{4}{|c|}{ Erosion } & \multirow{3}{*}{ Total } & \multirow{3}{*}{$\%$} \\
\hline & \multicolumn{2}{|c|}{$\begin{array}{c}\text { Erosion } \\
(\text { disease }+)\end{array}$} & \multicolumn{2}{|c|}{$\begin{array}{c}\text { No Erosion } \\
\text { (disease -) }\end{array}$} & & \\
\hline & $\mathbf{n}$ & $\%$ & $\mathbf{n}$ & $\%$ & & \\
\hline II (exposure +) & 82 & 51.2 & 78 & 48.8 & 160 & 100 \\
\hline I (exposure -) & 136 & 85 & 24 & 15 & 160 & 100 \\
\hline Total & 218 & 68,1 & 102 & 31,9 & 320 & 100 \\
\hline
\end{tabular}

meaning that erosion was less likely to occur in group II than in group I. In other words, mangosteen pericarp extract possessed protective effects.

In the current study, upon examination group II demonstrated gastric mucosal damage with a score of 6 . It was estimated that mangosteen pericarp extract could not preserve gastric mucosa integrity perfectly or mangosteen pericarp extract dosing was not optimal.

Moreover, Aspirin and other NSAIDs may lead to mucosal damage by several mechanisms: endothelial effect causing ischemia, direct toxicity from ion trapping which inhibits oxidative phosphorylation, and interruption of prostaglandin synthesis which impairs mucosal defense and repair. Ion trapping toxicity causes mitochondrial dysfunction as demonstrated from decreased stage-3 respiration, dehydrogenase activity, and transmembrane potential. ${ }^{7}$ Mitochondrial dysfunction leads to the prolific generation of reactive oxygen species (ROS) which in turn, causes cellular injury and increase opening of the permeability transition complex pores, eventually culminating in apoptosis. ${ }^{8}$ Additionally, ROS scavenging by other chemicals restores the mitochondrial respiration, dehydrogenase activity, and transmembrane potential, indicating restoration of mitochondrial function. These scavengers also prevent the activation of mitochondrial pathway of apoptosisby inhibiting caspase- 9 and caspase- 3 activities. ${ }^{7}$

Besides, xanthones present in mangosteen pericarp extract possess very good antioxidant capabilities and thus, could act as free radicalscavenging systems. ${ }^{4,8}$ At least 50 distinct xanthones have been identified present in the mangosteen pericarp at higher concentrations than in the aril or edible portion of the fruit. ${ }^{9}$ Diverse array of xanthone compounds open possibilities in search of the most potent xanthone.
The present study was consistent with a study performed by Nainwal et al. ${ }^{5}$ which showed that mangosteen pericarp extract displayed an antiulcerogenic effect at a dose of $500 \mathrm{mg} / \mathrm{kg}$ body weight. Nainwal et al. ${ }^{5}$ suggested that the antiulcerogenic effects were associated with the cytoprotective activity caused by the increased synthesis of mucus and/or prostaglandins.

Previous animal studies demonstrated that vitamin $\mathrm{C}$ reduced gastric mucosal damage induced by aspirin.They argued that these effects were due to vitamin $\mathrm{C}$ antioxidant activity by mechanisms involving preservation of gastric microcirculation, attenuation of lipid peroxidation and release of proinflammatory cytokines. Increased expression and activity of hemeoxygenase-1 (HO-1) played an important role in gastro-protection against NSAID by making cells more resistant to apoptotic death. ${ }^{10}$

Furthermore, different explanations from several studies indicated there were numerous possible mechanisms by which mangosteen pericarp extract could reduce the incidence of gastric mucosal damage. Further studies are needed to determine the mechanism of mangosteen pericarp extract in reducing gastric mucosal damage.

The primary limitation of this study was measurement bias incurred when determining the gastric mucosal damage scores based on Wattimena's criteria. Several studies used camera and image analyzer computer system to determine the gastric mucosal damage accurately and thus, could eliminate possible bias. .,10,11 $^{2}$ Further studies are recommended to use better parameters such as the lesion index for morphometric examination with aid of computer systems.

In conclusion, rats pretreated with mangosteen pericarp extract exhibit less gastric mucosal damage, in experimentally aspirin-induced ulcer. 


\section{References}

1. Yuan Y, Padol IT, Hunt RH. Peptic ulcer disease today. Nat Rev Gastroenterol Hepatol. 2006;3(2):80-9.

2. Ramakrishnan K, Salinas RC. Peptic ulcer disease. Am Fam Physician. 2007;76(7):1005-12.

3. Jainu M,MohanKV,DeviCS.Gastroprotective effect of cissus quadrangularis extract in rats with experimentally induced ulcer. Indian J Med Res. 2006;123(6):799-806.

4. Obolskiy D, Pischel I, Siriwatanametanon N, Heinrich M. Garcinia mangostana L.: a phytochemical and pharmacological review. Phytother Res. 2009;23(8):104765.

5. Nainwal P, Nanda D, Kalra K, Tripathi SM. Antiulcerogenic effect on the ethanol extract of the fruits of Garcinia mangostana on experimental gastric ulcer in rats. Int J Toxicol Pharmacol Res. 2010;2(1):6-9.

6. Wattimena JR. L-hypoprotenemie experimentale chez le rat, explotation pharmacocinetique du modele. These Doct'eur D'Etat Es Sciences Pharmacetiques. Montpellier: Faculte de Pharmacie, Universite Montpellier; 1982. p. 17-23.

7. Maity P, Bindu S, Dey S, Goyal M, Alam A, Pal $\mathrm{C}$, et al. Indomethacin, a non-steroidal antiinflammatory drug, develops gastropathy by inducing reactive oxygen speciesmediated mitochondrial pathology and associated apoptosis in gastric mucosa: a novel role of mitochondrial aconitase oxidation. J Biol Chem. 2009;284(5):305868.

8. Kumar V, Abbas AK, Fausto N, Aster JC. Robbins \& Cotran Pathologic Basis of Disease. 8th ed. Philadelphia: Elsevier Health Sciences; 2009.

9. Gutierrez-Orozco F, Failla ML. Biological activities and bioavailability of mangosteen xanthones: a critical review of the current evidence. Nutrients. 2013;5(8):3163-83.

10. Seleem HS, Ghobashy HA, Zolfakar AS. Effect of aspirin versus aspirin and vitamin c on gastric mucosa (fundus) of adult male albino rats. histological and morphometric study. Egypt J Histol. 2010;33(2):313-26.

11. Wang Z, Hasegawa J, Wang X, Matsuda A, Tokuda T, Miura N, et al. Protective effects of ginger against aspirin-induced gastric ulcers in rats. Yonago Acta medica. 2011;54(1):11-9. 